\title{
Terminal
}

Technologie de l'information, culture \& société

$127 \mid 2020$

Les groupes minoritaires et/ou marginalisés à l'ère numérique

\section{Vidéo en ligne et contestation politique radicale}

Entre intégration aux pratiques militantes et critique des plateformes

Online Video and Radical Political Protest: Embedded to Activist Practices or

Criticizing Online Platforms?

\section{Vincent Carlino}

\section{(2) OpenEdition}

Journals

\section{Édition électronique}

URL : http://journals.openedition.org/terminal/5806

DOI : $10.4000 /$ terminal.5806

ISSN : 2429-4578

Éditeur

CREIS-Terminal

\section{Référence électronique}

Vincent Carlino, «Vidéo en ligne et contestation politique radicale », Terminal [En ligne], 127 | 2020, mis en ligne le 20 avril 2020, consulté le 26 avril 2020. URL : http://journals.openedition.org/terminal/5806 ; DOI : https://doi.org/10.4000/terminal.5806

Ce document a été généré automatiquement le 26 avril 2020

tous droits réservés 


\title{
Vidéo en ligne et contestation politique radicale
}

\author{
Entre intégration aux pratiques militantes et critique des plateformes \\ Online Video and Radical Political Protest : Embedded to Activist Practices or \\ Criticizing Online Platforms?
}

Vincent Carlino

1 Le recours grandissant aux médias socionumériques dans les mobilisations sociales a tendance à souligner la portée démocratique du numérique. Afin de saisir les potentialités émancipatrices des technologies, il est nécessaire de contextualiser les usages des activistes. En effet, ceux-ci s'insèrent dans des contextes sociaux et politiques singuliers. Par exemple, la mobilisation sur les groupes Facebook est souvent identifiée comme point de départ de la révolution égyptienne de 2011 alors qu'elle repose sur une «base de protestation » préétablie dans la blogosphère (Faris, 2012, p. 102). Les mouvements contestataires reposent alors sur des usages politiques des médias sociaux par des groupes minoritaires et marginalisés. La vidéo occupe une place singulière dans cette mise en visibilité car elle permet de montrer au plus grand nombre des violences et des injustices subies, telles que des images de torture publiées par des blogueurs égyptiens. En 2011, le mouvement Occupy qui surgit en écho au Printemps arabe montre que la vidéo peut constituer un puissant moyen d'expression en diffusant des images d'assemblées réunissant de nombreux activistes. La diffusion vidéo en direct sur Internet devient « un symbole clé de la sophistication des pratiques médiatiques du mouvement » (Costanza-Chock, 2012, p.382). Elle n'est plus seulement utilisée pour dénoncer des situations, mais afin de mettre en scène la mobilisation et d'inciter les spectateurs à la rejoindre.

2 Cet article analyse l'usage de la vidéo par des militants écologistes opposés à l'enfouissement des déchets radioactifs dans la commune de Bure en France. Il porte plus précisément sur la période de mobilisation qui a débuté en juin 2016 au bois Lejuc, une zone de travaux du projet de Centre industriel de stockage géologique (Cigéo) qu'ils entendent occuper. À l'instar des activistes d'autres mobilisations dont celles citées 
précédemment, des militants ont investi les lieux pour ancrer la contestation sur le territoire. À la suite de la définition avancée par les coordonnateurs du dossier thématique, nous proposons de considérer que l'appartenance de ces militants à des réseaux militants perçus négativement par la culture dominante les mène à former un groupe marginalisé dans les espaces médiatiques dominants. Le « vidéo-activisme » qui se développe en 1980 vise à « lutter contre la censure et les effets d'agenda des médias mainstream par la production alternative d'images. » (Cardon et Granjon, 2013, p.100). Si cet engagement se retrouve à travers la vidéo en ligne, celle-ci ouvre de nouvelles interrogations. En effet, la vidéo en ligne occupe une place grandissante dans les pratiques des internautes, notamment via YouTube. Les vidéos contestataires se trouvent diffusées sur la même plateforme que d'autres contenus d'information et de divertissement. Si les vidéos contestataires des années 1980 se dressaient contre la télévision, le canal de diffusion des vidéos numériques n'incarne plus aussi nettement la rupture avec les espaces médiatiques dominants. L'objectif de l'article est d'explorer ce que les militants font de, et avec, la vidéo en ligne. Dans quelle mesure façonne-t-elle la mobilisation? En quoi les codes et les normes de production propres aux plateformes de diffusion, notamment YouTube, modifient-ils les modes d'engagement ? L'hypothèse avancée est que l'intégration et/ou la critique des codes des plateformes de vidéo en ligne permet aux groupes marginalisés de s'exprimer et de gagner en visibilité.

La méthode repose sur l'analyse d'un corpus de 24 vidéos en majorité réalisées entre 2016 et 2017 pendant la période d'occupation du bois Lejuc. Leur nombre a priori restreint s'explique par le caractère expérimental de leur production. Tournées par des amateurs aussi bien que des professionnels, ces vidéos sont marquées par leur caractère spontané visant à documenter cette période d'occupation devenue singulière dans la lutte antinucléaire à Bure. Notons que la priorité donnée à l'animation régulière du site Web «Plus Bure sera leur chute » peut expliquer ce faible nombre de vidéos. Si la focale est placée sur l'usage de la vidéo en ligne, l'analyse est aussi sensible à une approche esthétique attentive aux cadrages, mouvements de caméra, ainsi qu'aux textes et aux sons dans les vidéos. Des vidéos produites en partenariat avec l'Agence pour la gestion des déchets radioactifs (Andra) ont aussi été intégrées au corpus afin de rendre compte de l'appropriation des normes de YouTube par les promoteurs de Cigéo. Il s'agit des vidéos des YouTubeurs Anonimal ${ }^{1}$, Dave Sheik ${ }^{2}$, et Simon Puech ${ }^{3}$. L'étude repose aussi sur deux entretiens semi-directifs menés avec des militants ayant participé à la production de vidéos ${ }^{4}$. Ce faible échantillon s'explique par la dispersion des ex-occupants du bois Lejuc à la suite de l'expulsion du 22 février 2018. Les difficultés liées à la recherche de données numériques en partie effacées ou indisponibles sur le Web, ainsi qu'à la rencontre d'individus ayant rejoint d'autres luttes soulèvent une question de méthode. Ainsi, l'approche des technologies est ici " écologique » (Mabi et Gruson-Daniel, 2018) dans la mesure où leurs usages restent liés aux trajectoires des acteurs et à un contexte politique singulier. Les matériaux et données de recherche apparaissent le plus souvent comme des traces numériques qui rendent difficile leur identification et leur archivage par le chercheur (Bourdeloie et Chevret-Castellani, 2019). Dans un premier temps, nous proposons de situer la vidéo en ligne par rapport à l'histoire du mouvement contestataire contre Cigéo. Il s'agira notamment de déterminer en quoi la vidéo permet à une contestation radicale et marginalisée de s'exprimer. Ensuite, nous verrons que la conception de vidéos s'inscrit dans des pratiques militantes qui trouvent de nouvelles manières de "se réaliser " 
grâce à elle. Enfin, nous étudierons comment le vidéo-activisme s'inscrit dans une guerre d'images avec les promoteurs du nucléaire qui se joue notamment à travers la connaissance des normes de production des plateformes.

\section{L'expression d'une opposition radicale marginalisée}

4 Le mouvement contestataire qui s'est installé à Bure en 2016 s'inscrit dans la continuité d'une opposition locale apparue depuis plus de vingt ans. Plus largement, les opposants contestent les décisions politiques prises dès la fin des années 1970 visant à résoudre la question de la gestion des déchets radioactifs les plus dangereux. C'est en 1979 que le Commissariat à l'énergie atomique (CEA) crée en son sein l'Agence pour la gestion des déchets radioactifs (Andra). Son objectif est de gérer les déchets radioactifs générés par la filière électronucléaire française. Parmi eux, les déchets radioactifs dits de haute (HA) ou de moyenne activité à vie longue (MA-VL) constituent le principal problème. Bien qu'ils ne constituent que $3 \%$ du volume total des déchets, ils concentrent 99,8\% de la radioactivité totale (Andra, 2019, p.15). Afin de confiner ces déchets les plus dangereux, l'État français lance en 1987 un programme d'investigation dans quatre départements: l'Aisne, l'Ain, le Maine-et-Loire et les Deux-Sèvres. Très vite, une opposition virulente se dresse devant les ingénieurs et les représentants politiques en visite pour étudier les propriétés du sol sans concertation préalable avec les populations locales. Des gendarmes répriment les violences, ce qui contraint le Premier ministre Michel Rocard à évacuer les sites en prononçant un moratoire. En 1991, le rapport du député Christian Bataille aboutit à une loi « relative aux recherches sur la gestion des déchets radioactifs». Celle-ci entérine un «nouveau mode de gouvernement» (Patinaux, 2017, p.89) fondé sur l'évaluation de la qualité des recherches sur les déchets radioactifs.

Progressivement, le sous-sol argileux de la Meuse et de la Haute Marne est envisagé. Malgré l'opposition de la population et d'élus locaux, l'Andra est chargée de construire un laboratoire de recherche à Bure. Dans les faits, l'Andra y mène des recherches en vue de construire Cigéo quelques kilomètres plus loin. En 2005, la Commission nationale du débat public (CNDP) organise un débat aboutissant à la loi de 2006 fixant le "stockage profond réversible» comme "solution de référence». Les militants d'associations locales deviennent des "citoyens-enquêteurs" (Carlino, 2019) et s'opposent à Cigéo sur leurs sites Web respectifs.

6 En 2013, la CNDP lance une concertation pour « informer le public sur le projet ${ }^{5}$ » Cigéo. Les associations décident alors un boycott collectif car ils jugent que cette concertation vise à obtenir leur consentement. Les réunions sont tendues : les responsables sont accueillis sous les sifflets et les échanges ne peuvent plus se tenir. Certains militants voient ce conflit comme la preuve de l'inefficacité des actions plus policées et institutionnelles du «militantisme de dossier » (Ollitrault, 1996) qui ne parvient pas à faire reculer Cigéo.

7 En refusant le cadrage du débat public, ils se trouvent marginalisés par les instances officielles. On peut alors se demander quels sont les moyens à leur disposition pour s'exprimer en dehors des procédures qu'ils contestent (Blondiaux, 2008, p.136). De plus, les militants sont marginalisés du fait de leur critique radicale de l'État et du capitalisme. Ils se situent à la marge du répertoire d'action antinucléaire ${ }^{6}$ jusque-là fondé sur la contre-expertise (Topçu, 2006), dirigeant leur action vers l'occupation de 
«contre-lieux» (Lussault, 2017) qui matérialisent leur volonté de développer des formes de vie collective alternatives à celles des acteurs majoritaires qui commandent Cigéo. Bien qu'il soit pacifiste, ce mode d'action n'exclut pas le recours à l'affrontement physique. À Bure, les tensions se sont cristallisées au bois Lejuc, zone stratégique pour l'éventuelle construction de Cigéo. En s'y installant, les opposants ont rendu tangible la volonté de l'Andra d'occuper ce territoire et d'avoir recours à la force publique pour repousser quiconque voudrait l'en empêcher. Aussi, la présence des militants a rendu visibles plusieurs individus déterminés à repousser l'Andra pour préserver la forêt. Ce conflit est singulier par sa dissymétrie : ceux qui manifestent leur désaccord semblent disposer de moins de ressources (humaines, financières) que leurs adversaires, et paraissent minoritaires. Ce dernier aspect constitue d'ailleurs le troisième facteur de marginalisation des opposants radicaux à Cigéo. En effet, certains estiment subir une répression voire des violences policières non seulement au cours de manifestations, mais aussi du fait de leur engagement militant (écoutes, perquisitions, patrouilles).

La contestation se compose d'une multiplicité d'arènes qui insistent chacune sur une dimension particulière (judiciaire, financière, politique, éthique, etc.). Cet éclatement peut être pensé en lien avec les inégalités sociales. Selon Nancy Fraser (2001, p.133), la sphère publique se compose d'une pluralité de contre-publics : nationalistes, paysans populaires, femmes de l'élite, classe ouvrière. Pour la chercheuse, ces groupes minoritaires constituent des « contre-publics subalternes » engagés dans la lutte pour formuler leur propre interprétation de leurs identités, leurs intérêts et leurs besoins (ibid., p.138). Quels moyens les militants antinucléaires emploient-ils pour constituer un tel groupe subalterne? La vidéo en ligne constitue un moyen de prendre part au débat sans se conformer aux normes délibératives. Internet est souvent présenté comme étant adapté pour contourner les médias dominants et faire vivre un espace public oppositionnel (Negt, 1972) qui donne toute leur place aux groupes minoritaires (Granjon et al., 2017, p.133). L'hypothèse avancée est que la créativité et la manipulation d'outils inhérentes à la réalisation de vidéos correspond d'autant plus à "l'expérience sensible, liée au corps, au travail vivant et à la culture collective " (Neumann, 2018) qui caractérise l'espace public oppositionnel.

\section{Lutter par la vidéo}

9 Les militants n'ont pas attendu d'occuper le bois Lejuc pour capter des images de la contestation. Jacqueline et Alain Riès ont réalisé «Tous n'ont pas dit oui » (2007), le premier documentaire sur la lutte contre les déchets radioactifs dans la Meuse. Quelques années plus tard, le film " 100000 ans sous nos pieds» de Dominique Hennequin documente les risques liés à l'enfouissement des déchets radioactifs. En 2015, les frères Sébastien et Aymeric Bonetti racontent leur immersion à la « Maison de résistance ", une habitation collective à Bure. Centré sur l'observation des pratiques militantes, leur film préfigure les vidéos produites lors de l'occupation du bois Lejuc car la production de vidéos est immergée dans l'action collective.

10 Les recherches sur la vidéo en ligne concernent surtout la communication politique. Les travaux soulignent la place grandissante de YouTube dans les campagnes électorales (Theviot, 2018; Dias da Silva, 2015 ; Yanoshevsky, 2009). YouTube est aussi étudié pour saisir la participation d'individus "étrangers au militantisme » dans le débat politique (Babeau, 2014). La vidéo s’intègre parmi des études qui soulignent le 
rôle de plus en plus important des contenus visuels dans la communication politique en ligne (Towner et Muñoz, 2018). En outre, les nouveaux formats tels que les vlogs permettent une certaine libération de la parole dans les groupes minoritaires (Balleys, 2019). Si les technologies de l'information et de la communication ont été largement étudiées dans l'organisation de milieux militants (Granjon, 2003; Sedda, 2015), l'exploration de leurs effets dans des groupes contestataires marginalisés reste encore à écrire. L'exclusion d'espaces de délibération politique est fréquemment soulignée chez les groupes minoritaires faiblement politisés et/ou vulnérables (Wamé, 2018). Elle se pose aussi aux militants antinucléaires radicaux davantage politisés. En effet, la vidéo en ligne contribue à façonner voire à renforcer l'engagement des activistes. Sa diffusion sur des plateformes numériques implique une réflexion sur le rapport à l'outil et la compatibilité de certains codes utilisés avec les valeurs des acteurs. Cet article explore donc le champ de recherche encore peu investi des effets politiques internes de la vidéo dans les mouvements sociaux. L'objectif est de souligner comment la production et la diffusion de vidéos sur les plateformes numériques façonne ces mouvements.

La convergence de plusieurs militants vers le bois Lejuc en 2016 offre un terrain propice à l'analyse de ces effets. Leur combat n'est pas seulement dirigé contre le projet, mais contre le modèle de société qui le réalise. Pour eux, la critique de Cigéo est considérée comme allant d'elle-même, le point de départ d'une contestation sociale plus vaste. Il s'agit là d'un trait commun aux mobilisations sociales contemporaines marquées par leur convergence. D'ailleurs, plusieurs des occupants de la forêt meusienne proviennent du mouvement Nuit Debout (Les Temps Modernes, 2016). L'occupation durant trois semaines de l'été 2016 souhaite promouvoir des modes de vie et de développement alternatifs à ceux de l'Andra. La forêt devient un «contre-lieu » dont on souhaite « conserver l'authenticité par les modes de cohabitation choisis et promus par ceux qui entendent [la] protéger en y demeurant. » (Lussault, 2017, p.229). À travers leur occupation, les activistes réfléchissent à des manières d'habiter le territoire qu'ils estiment plus respectueuses. Ils interrogent aussi les outils numériques. Sans vouloir les rejeter totalement, ils questionnent leurs usages pour déterminer si les technologies s'alignent ou non avec leurs valeurs :

«Il faut toujours être vigilant.es pour garder un cap juste, éviter le sensationnalisme, renouveler notre ton, ne pas confisquer la parole à des personnes subissant des oppressions, ne pas devenir des geeks des réseaux sociaux etc ${ }^{7}$. "

13 Ce tâtonnement met en tension l'exploitation des potentialités expressives des outils numériques avec la recherche $\mathrm{du}$ " ton » auquel ils se réfèrent, ainsi que le risque qu'ils détournent les individus de leur volonté première pour se consacrer uniquement à leur communication en ligne et devenir « des geeks », selon les mots employés sur leur site Web. La vidéo répond à cette quête d'expérimentation car elle ne dépend pas totalement des plateformes où elle est diffusée ${ }^{8}$. En effet, les tweets de 280 caractères prennent tout leur sens lorsqu'ils sont publiés sur Twitter. Le design du réseau socionumérique qui se retrouve à travers le système de «j'aime », mots-clics, retweets, réponses, threads, timeline, etc., donne toute leur portée sémiotique aux contenus (Bonaccorsi, 2013 ; Candel et Gomez-Mejia, 2017). Si, comme nous le verrons, YouTube tend à opérer ce même mouvement pour la vidéo, celle-ci demeure relativement indépendante en tant que support, dans la mesure où elle peut être stockée et visualisée en des lieux multiples, en ligne ou non. 


\section{Au-delà du potentiel mobilisateur : filmer comme on lutte} détermination des opposants face à l'Andra pour la première et aux forces de l'ordre pour la seconde. Relativement courtes ( $3 \mathrm{~min} 17 \mathrm{~s}$ et $4 \mathrm{~min} 11 \mathrm{~s}$ ), elles partagent des caractéristiques communes. Toutes deux montrent la forêt vue d'en haut à travers des images capturées à l'aide d'une caméra de type GoPro fixée sur un grimpeur qui descend en rappel dans la forêt depuis une cabane nichée en haut des arbres. La forêt est aussi montrée depuis le point de vue des militants. Les différentes séquences suggèrent le bois Lejuc sans que celui-ci soit dévoilé dans sa totalité. Ce choix traduit une volonté de montrer la forêt de l'intérieur. On y voit le bois Lejuc composé d'arbres très hauts (impression renforcée par l'utilisation de la contre-plongée), de cabanes juchées sur leurs branches, de barricades et d'individus qui s'y promènent. Dans un second temps, les deux vidéos mettent en scène leur lutte avec l'adversaire. La première révèle la présence d'une barricade défensive composée de plaques de béton utilisées autrefois par l'Andra pour tenir les opposants à l'écart ${ }^{9}$. Le combat est rapidement tourné en dérision car il s'effectue avec des lancers de boules de neige vers la caméra. Il n'est pas aisé de fournir une interprétation de cette séquence car on peut supposer que la caméra prise pour cible est tenue par un militant antinucléaire. Difficile donc d'affirmer que c'est l'Andra qui est attaquée, ce que laissent pourtant penser les slogans déclamés: «Dégagez, dégagez ", "Andra dégage, résistance et affouage ! ". La vidéo ne donne donc pas toutes les clés de lecture et de compréhension. Le spectateur peut avoir l'impression de devoir se rendre sur place pour mieux comprendre la vidéo, mais surtout les pratiques de lutte qui l'entourent. 
Figure 1 : Capture d'une séquence du premier teaser mettant en scène une défense de barricade dans le bois Lejuc

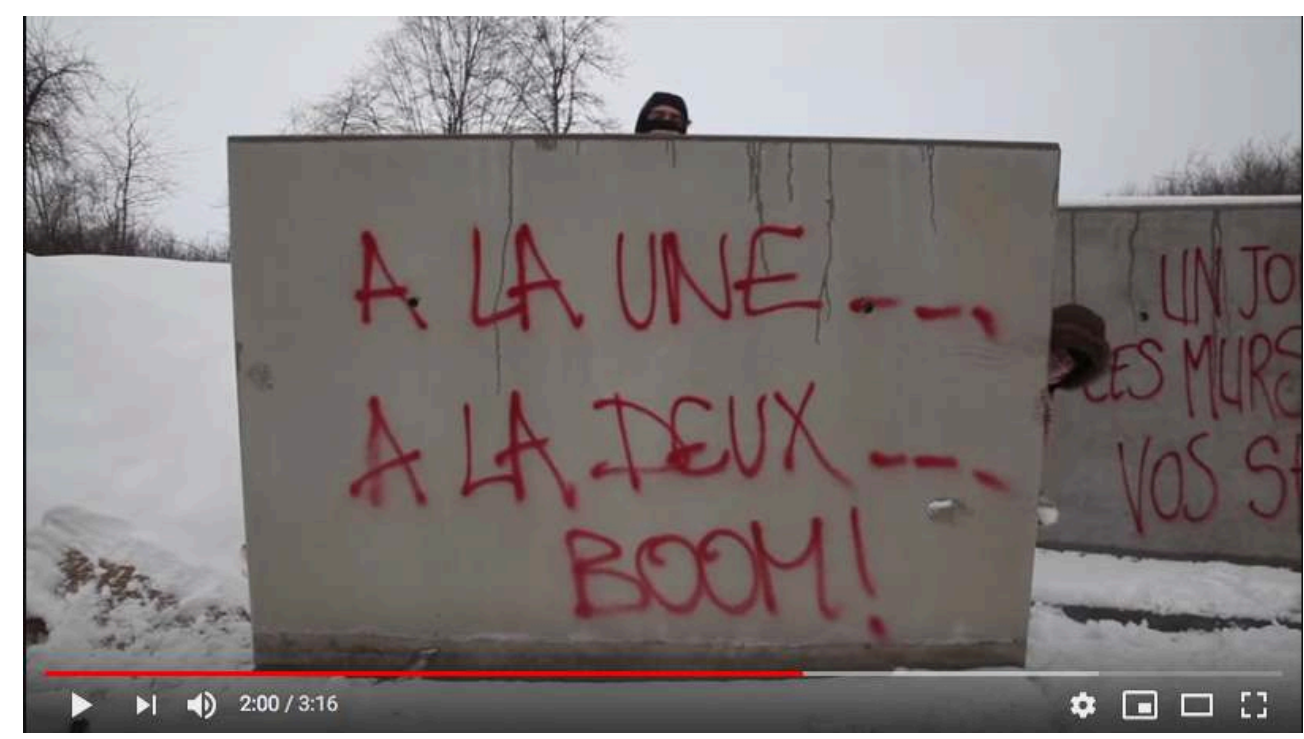

Dans la seconde vidéo, ce sont les forces de l'ordre qui sont prises pour cible à travers une chanson dont les paroles demandent «À quoi pensent les flics?». Sur le plan rhétorique, ces teasers se veulent le reflet de l'occupation de terrain. Dans les deux cas, la vidéo s'achève sur un texte en surimpression qui invite à rejoindre le mouvement sur place, comme pour découvrir la suite du film. Ainsi, la vidéo s'inscrit dans la continuité des capacités de mobilisation des outils numériques, déjà observées par ailleurs (Badouard, 2013). En tant que moyen de communication, la vidéo s'affirme surtout comme une manière de faire la lutte parmi d'autres activités dans le bois Lejuc. Cela se remarque notamment à travers une série de références à la zad de Notre Dame des Landes au moyen de codes esthétiques singuliers. Précisons que les zad désignent une forme de contestation apparue en 2007 et fondée sur l'occupation permanente ${ }^{10} d^{\prime}$ 'un site, sujet de la contestation, et la mobilisation de militants altermondialistes (Subra, 2017, p.12). La bande-son du film «Le bon, la brute et le truand» d'Ennio Morricone utilisée dans le premier teaser est une référence au western tourné sur la zad. De même, la musique bretonne évoque l'ouest de la France. Selon une militante interrogée, les barricades font aussi écho à celles de Notre-Dame-des-Landes, ainsi que celles de l'affrontement dans la neige. Ces codes sont directement adressés aux habitués des luttes sociales et environnementales et échappent à la majorité des autres qui se focaliseront sur l'appel à mobilisation. La vidéo établit donc une certaine convergence entre des luttes qui partagent un projet politique commun.

\section{Réaliser des films, se réaliser}

17 Si l'occupation caractérise les mobilisations contemporaines, avec notamment Occupy, les zad, Nuit Debout ou les Gilets jaunes, leurs dynamiques internes restent encore peu connues. Sans prétendre contribuer à leur compréhension ici, soulignons que les deux personnes interrogées ont insisté sur "l'effervescence" du bois Lejuc. Vivre en permanence sur un même lieu avec des inconnus implique que chaque action de la vie quotidienne soit lié à la lutte et, par là, acquiert une portée politique. Perçue comme hors du temps et des contraintes de la vie urbaine, l'occupation collective brouille les 
repères individuels et opère une profonde remise en question chez des individus qui (re)pensent leur place dans la société. Cette fragilité des "carrières occupantes " (Dechézelles, 2017, p.96) souligne la marginalité d'individus associés à des «styles de vie déviants" (ibid.). Du point de vue des technologies numériques, l'habitation collective semble déterminante. En effet, ce basculement des repères individuels alimente un élan créatif où la vidéo joue un rôle central. Il ne s'agit pas seulement de réaliser des supports de communication, mais de se réaliser à travers eux. Souvent, les vidéos émergent d'une volonté d'expression individuelle qui est ensuite soumise à une discussion collective visant à déterminer si elle est représentative de la lutte ou non.

La vidéo devient alors un moyen de trouver sa place dans la mobilisation pour des individus marginalisés. La conception de vidéo permet alors de tisser du commun. Par exemple, la chanson dans un des teasers s'achève par des hésitations et des rires qui auraient pu être retirés au montage final. Ceux-ci ont été laissés, comme pour refléter une certaine authenticité, mais aussi pour rendre compte du caractère artisanal des productions qui pourrait traduire une certaine complicité. La captation de manifestations telles que la marche nocturne aux flambeaux exprime également cette tension entre le soin apporté à l'image et le bricolage visible dans des séquences ralenties, floues à l'écran.

Par cet usage de la vidéo, le numérique devient un outil d'écriture de la lutte. Alors que la haute définition s'est démocratisée avec les technologies et les connexions haut débit, le numérique est utilisé pour redonner du grain et des "défauts » à l'image. Il s'agit de retrouver un rapport physique, quasi charnel, semblable à la vidéo analogique. Des militants se sont d'ailleurs essayés à la caméra super $8^{11}$ dont la pellicule contraint les modes d'expression. Ce rapport analogique au numérique vise à émanciper les individus par le processus créatif. La vidéo ne sert pas seulement à mobiliser, mais aussi à favoriser l'expression des membres des groupes minoritaires. Lorsque des victoires sont obtenues sur le terrain, les films insistent sur la joie des individus comme si leur engagement était total et leur permettait de trouver une place dans la société. Par exemple, lorsque les militants ont abattu le mur érigé par l'Andra en 2016, ces derniers l'ont tourné en dérision dans une vidéo qui les présentent comme des plaisanciers de "Bure les bains », utilisant les plaques de béton en guise de chaises longues.

\section{De l'auto-défense médiatique au témoignage}

Parce qu'elle s'intègre aux pratiques contestataires, la vidéo est indissociable de cellesci. Il ne s'agit plus seulement de déterminer son potentiel de mobilisation, mais la manière dont elle contribue à façonner la contestation. Si elle peut être vecteur d'émancipation individuelle, la vidéo est aussi un moyen de se défendre face aux oppressions. La médiatisation du mouvement constitue une menace contre laquelle les opposants entendent agir. En effet, leur occupation est la première du genre dans la Meuse et la Haute-Marne. Certes, les antinucléaires sont présents sur place depuis 2004 dans une habitation collective, la «Maison de résistance " (d'Allens et Fuori, 2017, p. 100). Cependant, l'occupation hors les murs d'une forêt représente un potentiel événement médiatique, d'autant que les zad cristallisent l'attention à cette époque. Face à la venue de médias locaux et nationaux, les militants constituent un groupe dédié à la communication et aux relations presse: l'« Automédia ». Ce collectif vise autant à contrôler les entrées et sorties d'information médiatique qu'à médiatiser la 
lutte. Sur Internet, les activistes qui commentent un événement expriment aussi leurs émotions et justifient ce qui fonde leur point de vue (Mabi, 2016 ; Sebbah, 2017). Dans les vidéos, cette subjectivité est surinvestie si bien qu'elle l'emporte sur la cause. En effet, aucune d'entre elles n'entre dans le détail de la controverse des déchets radioactifs, mais toutes s'arrêtent sur le récit de l'occupation collective du bois Lejuc. Dans le même temps, la vidéo est conçue comme une arme d'autodéfense médiatique permettant de symétriser les relations avec les journalistes :

"Quand c'était un entretien filmé, on filmait, quand c'était un entretien enregistré, on enregistrait. Ce qui impose une certaine pression au journaliste. Si jamais on a la sensation que l'entretien n'est pas le reflet de la réalité, on a l'original. On peut très bien republier sans coupe la totalité de l'entretien. [...] Par le biais des réseaux sociaux, on a acquis la possibilité d'être un véritable média. On a la capacité d'être des médias au même titre que les journalistes en termes d'audience. " (Entretien avec un membre de l'Automédia, 26/5/2019).

Même lorsqu'elle n'est pas diffusée, la vidéo détient une place singulière dans la lutte. D'une part, les activistes font preuve d'une méfiance à l'égard des journalistes mais, d'autre part, ils présentent la captation vidéo des interviews avec ceux qui les interrogent comme le reflet d'une objectivité absolue. Or, toute vidéo suppose un cadrage et une focalisation sur des éléments au détriment d'autres, si bien qu'on ne peut pas assimiler ces images à celles d'une caméra de vidéosurveillance par exemple. Ici, la vidéo a une fonction sociale puisqu'elle permet de rééquilibrer une relation jugée dissymétrique. La critique des médias ne repose pas sur un rejet total du champ médiatique, mais sur une relation de confiance avec les journalistes pour que leurs propos soient le plus fidèlement transcrits. Ainsi, les militants n'excluent pas de recourir aux médias numériques car ils les conçoivent comme étant au service de leur parole, sans nécessité d'entrer dans les logiques des plateformes de diffusion.

Cette représentation de la vidéo en tant que reflet du réel se retrouve avec l'utilisation de caméras embarquées de type GoPro. Leur enregistrement en continu alimente la croyance qu'elles peuvent tout capter. Ces images brutes ne sont pas destinées à être diffusées, mais à protéger des personnes qui pourraient faire l'objet de poursuites à la suite des actes commis dans la manifestation. Cet usage correspond aux pratiques de "sous-veillance " développées avec l'adoption de dispositifs de captation embarqués (wearable computing). Légères et souvent portées sur une partie du corps, les caméras sportives correspondent à ces outils. Leur usage consiste à surveiller l'adversaire « d'en bas ", du point de vue de ceux qui se sentent opprimés. Toutefois, la captation seule ne suffit pas. Son efficacité dépend des "technologies médiatiques qui diffusent le message » (Mann et Ferenbok, 2013, p.24), si bien que la "sous-veillance » articule les technologies de captation aux logiques des médias traditionnels et numériques.

La confrontation entre des militants et un ingénieur de l'Andra le 31 janvier 2017 fournit un exemple d'une telle médiatisation. Une vidéo montre l'ingénieur versant de l'essence au pied d'activistes barrant un engin de chantier (Fig. 2). La séquence longue d'une minute montre l'individu essayant de retirer la barricade. Après être sorti du cadre, celui-ci revient avec une bouteille en plastique contenant un liquide, l'ouvre et en respire le contenu avant de le déverser au sol. La voix du cadreur se fait alors entendre : «C'est classe, ça va faire très beau sur la caméra! ». Publiée sur YouTube, la vidéo comptabilise plus de 20000 vues. Elle a ensuite été diffusée dans un reportage de la chaîne de télévision locale France 3 Lorraine $^{12}$. 
Figure 2 : Capture de la séquence montrant un employé de l'Andra versant de l'essence à proximité de militants dans le bois Lejuc

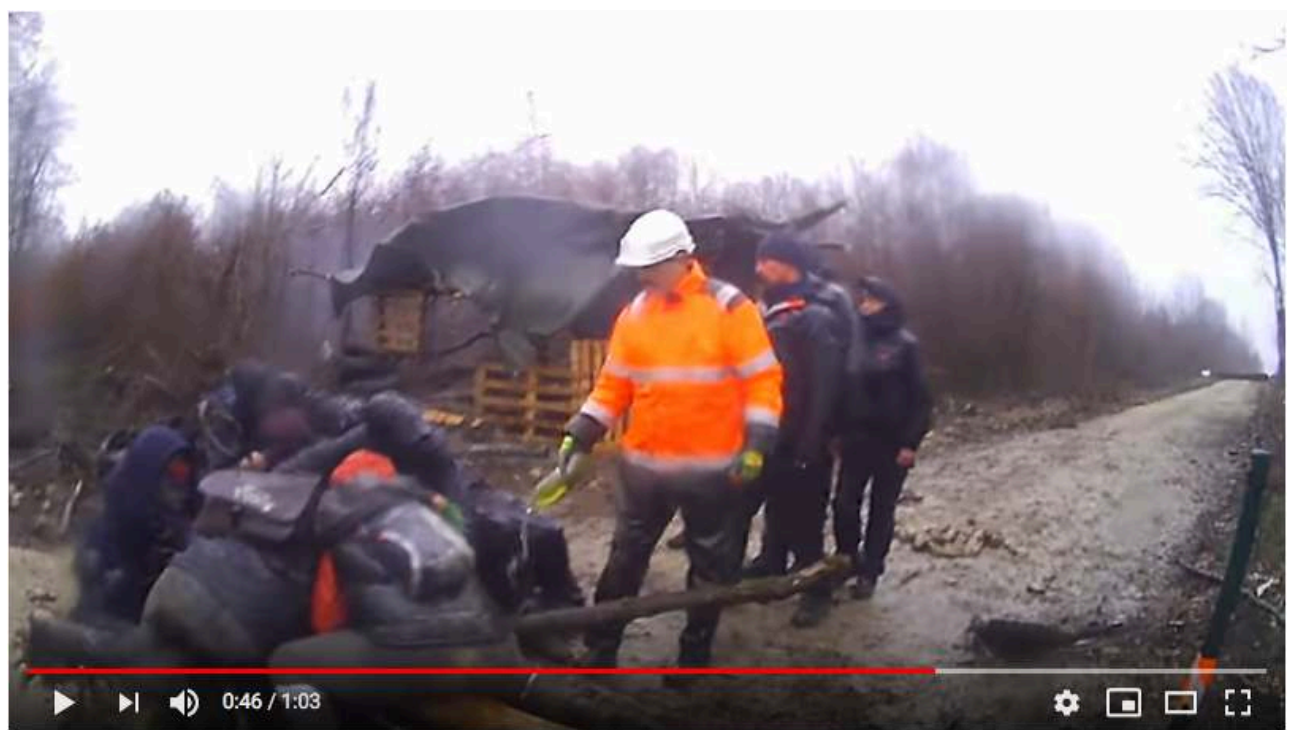

Cette vidéo souligne l'usage stratégique et engage une guerre d'images. En effet, la «sous-veillance » vise à contrer un discours qui présente les activistes comme des écologistes radicalisés. L'objectif est d'offrir des "représentations d'eux-mêmes " différentes des images médiatiques dominantes (Cuny et Nez, 2013, p.19). En occupant le territoire, les militants côtoient de fait des employés de l'Andra eux aussi présents dans le bois pour mener des travaux préparatoires. Les filmer permet de dévoiler des pratiques qu'ils jugent violentes et invisibles. Par le jeu de la captation d'images brutes diffusées en ligne puis reprises par une chaîne de télévision, les activistes rendent visibles ce qu'ils étaient jusque-là seuls à observer.

Le développement de vidéos de témoignage s'inscrit également dans ce conflit d'images. Alors que l'Automédia refuse toute incarnation du mouvement, plusieurs militants décident de témoigner à visage découvert de la répression qu'ils subissent. La chaîne YouTube " Paroles de malfaiteur-e-s » se créée alors pour montrer comment les militants vivent malgré les actions judiciaires dont ils font l'objet. Les quatre vidéos publiées sont tournées chez les interrogés, en plan fixe, et durent environ dix minutes. Après une courte présentation biographique, les individus témoignent de leur «vie sous contrôle judiciaire » et des écoutes policières. Le témoignage devient alors une façon de contribuer au débat public sans se conformer aux normes de la délibération (Young, 2002). Les témoins montrent que l'enfouissement des déchets radioactifs n'est pas uniquement scientifique et technique, mais qu'il porte une certaine idéologie qui, lorsqu'on s'y oppose frontalement, marginalise les individus dans la société en réduisant leurs droits (limitation des contacts entre militants, interdiction de territoire, écoutes téléphoniques, etc.).

Selon un membre de l'Automédia, le mot d'ordre de ce groupe est de « construire des choses qui ressemblent » aux militants. Les médias ne sont pas jugés pour ce qu'ils sont, mais pour leur capacité à retranscrire la lutte. L'Automédia se conçoit donc comme la faculté de témoigner sur l'action, laquelle peut autant se trouver dans des médias généralistes comme dans d'autres qui se qualifient d' "alternatifs ». Cependant, cela 
n'empêche pas la réflexion sur les plateformes numériques lorsque les individus décident de publiciser leur discours par eux-mêmes.

\section{Critique et intégration des normes des plateformes}

La diffusion de vidéos sur Internet suppose de recourir à des outils qui permettent de les héberger et de les lire à distance. Cette logique qui a dominé les débuts de la vidéo en ligne est aujourd'hui mise en cause par les plateformes qui se pensent comme des diffuseurs de contenu. L'objectif est que l'utilisateur reste sur la plateforme après y avoir déposé du contenu pour développer son audience. Les spectateurs se trouvent quant à eux happés par la recommandation de contenus après le visionnage d'une vidéo. Après son rachat par Google, YouTube se pose comme un média social. Le virage dans son modèle économique met l'accent sur les user generated content (UGC), c'est-àdire les contenus produits par les utilisateurs de la plateforme, souvent considérés comme des « professionnels amateurs » (Flichy, 2010).

L'enjeu consiste à réguler cette production de contenus de sorte à la professionnaliser et à la rendre compatible avec la publicité (Kim, 2012). Cette régulation s'exprime notamment par "des normes d'action, une architecture technique et des discours d'accompagnement (également normatifs)» (Bullich, 2015, p.29). Ces normes redéfinissent les contours de la vidéo à mi-chemin entre production amateur et marchande (Snickars et Vonderau, 2009, p.11). Plus précisément, elles résultent d'un hiatus entre la communication de YouTube qui valorise les UGC et sa stratégie commerciale visant à négocier des droits d'auteurs dans l'industrie médiatique. Si les agences publicitaires acceptent de diffuser des annonces autour de contenus industriels traditionnels (bandes annonces, clips musicaux, etc.), elles sont plus frileuses quant aux contenus communautaires qui dépassent les limites de la culture médiatique dominante (Andrejevic, 2009, p.413).

On peut alors se demander dans quelle mesure ces normes de production de contenus sont compatibles avec les valeurs des activistes. Ce questionnement revient à interroger les limites de la contestation radicale par la vidéo en ligne. Jusqu'où les militants acceptent-ils de se conformer à des normes qui ne sont pas en alignement avec leur engagement politique?

Deux normes semblent particulièrement mettre à l'épreuve l'opposition radicale. D'abord, certains codes esthétiques et graphiques sont récurrents chez les YouTubeurs. Sur YouTube, la vidéo existe avant le visionnage, c'est pourquoi la plateforme incite ses utilisateurs à soigner tout ce qui l'entoure de façon à maximiser ses chances d'être $v \mathrm{ve}^{13}$ (Fig. 3). Les titres sont particulièrement travaillés de manière à inciter l'utilisateur à découvrir le contenu. Les YouTubeurs mettent en avant des questions auxquelles ils sont censés répondre : "Comment préparer le futur? ", «Qu'est-ce qu'il y a après le nucléaire ? ", «L'énergie nucléaire : utile ou dangereux ? ». Ils livrent aussi leur point de vue, ce qui encourage à regarder le contenu pour renforcer ou non ses opinions préexistantes : "Vos factures EDF vont exploser : le vrai coût du nucléaire ", «Et tout le monde s'en fout : les déchets ». D'autres mettent en avant une aventure personnelle: "J'ai approché des déchets radioactifs ", "Exploration de Tchernobyl dans la zone radioactive ", "Dans les zones radioactives de Fukushima ». Les vignettes qui accompagnent ces contenus renforcent la présence du créateur qui incarne le discours et se pose comme un acteur à part entière. 
Figure 3 : Illustration et titre de la vidéo du YouTubeur « Anonimal » sur les déchets radioactifs

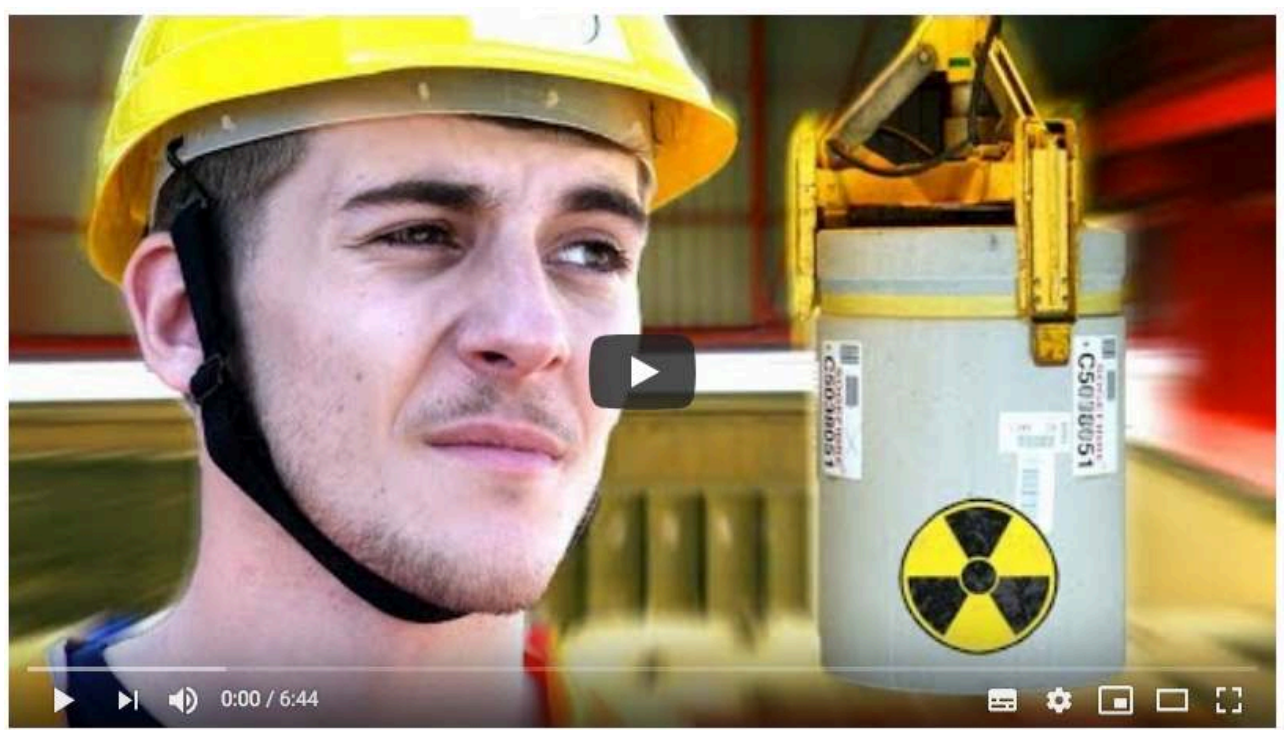

J'AI APPROCHÉ DES DÉCHETS RADIOACTIFS

La deuxième norme concerne le rythme de publication des vidéos. En effet, l'algorithme de recommandation se fonde en partie sur la publication régulière de contenus, si bien que les créateurs sont incités à suivre un rythme de production de façon à maximiser leur visibilité.

Les stratégies marketing de contenus deviennent un nouvel enjeu pour que les vidéastes gagnent en popularité. D’ailleurs, YouTube est devenu un lieu stratégique pour l'opinion publique sur Internet, ce qui explique que l'Andra a aussi investi la plateforme en finançant des partenariats avec plusieurs influenceurs (Frau-Meigs, 2017) pour se rendre visible auprès d'une audience plus jeune ${ }^{14}$. Sur YouTube, la présentation de soi ne se fait pas seulement à travers le contenu, mais aussi à travers la stratégie déployée pour le promouvoir ${ }^{15}$. A priori, cette logique et la lutte radicale sont antinomiques. Pourtant, elles s'inscrivent toutes les deux dans des «cultures expressives " (Allard, 2007) où l'individu devient son propre média. Le recours à YouTube n'est donc pas simplement technique. Il soulève des enjeux politiques en prise avec l'action collective :

44 «Il n'y avait pas une recherche vis-à-vis de YouTube. Dans tous les cas on ne partait pas de l'outil. L'outil doit juste être le vecteur, mais jamais la fin en soi. » (Entretien avec un membre de l'Automédia, 26/5/2019).

La mobilisation et les individus qui la composent déterminent l'usage de la plateforme, et non l'inverse. Ainsi, les militants utilisent les médias sociaux dès qu'ils les jugent cohérents avec leur engagement. Ce jugement s'opère par une discussion collective où les individus débattent pour déterminer si les contenus les reflètent, dans un souci d'inclusion. Si cette méthode a été éprouvée pour les contenus à proprement parler, elle reste à développer au sujet des plateformes. Jusqu'ici, le choix retenu est de diffuser le message sur un maximum de plateformes pour convenir à toutes les habitudes de visionnage ${ }^{16}$. La responsabilité politique repose donc sur le spectateur et non sur les militants qui justifient leur présence sur YouTube par commodité pour leur public. La question de la critique des normes marchandes des Gafam (Smyrnaios, 2017) 
n'est pas posée de front alors qu'elle semble pourtant inévitable, d'autant qu'elle fait écho à leur critique anticapitaliste.

\section{Conclusion}

36 À l'issue de cette réflexion, la vidéo en ligne apparaît comme un élément essentiel affirmant l'identité de militants radicaux marginalisés. En effet, leur production conduit les acteurs à questionner les valeurs politiques à défendre, lesquelles sont mises à l'épreuve des plateformes numériques et de leurs modèles économiques.

En premier lieu, il convient de ne pas négliger l'échelle individuelle pour éviter de focaliser exclusivement l'attention sur le potentiel de mobilisation des médias numériques. Si la vidéo est utilisée pour accroître la mobilisation, elle est surtout un moyen d'expression voire d'émancipation individuelle pour des individus minoritaires qui interrogent leur place dans la société. Les potentialités créatives de la vidéo montrent qu'elle peut être un moyen de prendre part au débat sans adopter les normes délibératives des espaces majoritaires fondées sur l'argumentation rationnelle. Cette réalisation des individus par la confection de vidéos ne signifie pas que celles-ci s'adressent uniquement à celui qui les fabrique. Au contraire, le processus de création est intégré aux autres pratiques militantes, si bien qu'il mêle professionnels du film et amateurs, mais repose aussi sur un certain «bricolage» qui renvoie à une forme d'authenticité.

38 Cette intégration de la vidéo au sein des actions collectives traditionnelles soulève des limites. En effet, la production dépend des individus engagés dans la contestation et de leur propre rapport aux médias numériques. Si la vidéo constitue un potentiel outil émancipateur pour les groupes marginalisés, ces derniers pensent encore leur diffusion sur le Web d'un point de vue technique. Or les plateformes de diffusion de contenu soulèvent des enjeux économiques et sociopolitiques forts qui gagneraient à s'intégrer aux réflexions. Il ne s'agit pas nécessairement de focaliser l'attention sur les Gafam et notamment YouTube qui occupe une position dominante sur le marché, mais d'identifier les portées politiques des plateformes à disposition : commerciales (Viméo, YouTube), libres de droit (Peertube) et militantes (Rise Up, Indymedia). Une telle cartographie permettrait de pratiquer un vidéo-activisme "situé » conscient des potentialités et limites de chaque plateforme, comme cela se pratique déjà avec les journalistes.

39 Enfin, il est aussi nécessaire d'interroger ce que les activistes peuvent faire aux médias numériques. Les formes de mobilisation mettent à l'épreuve les normes de fonctionnement des plateformes en ligne. La créativité assumée et envisagée comme vecteur d'émancipation peut contribuer à mettre en cause les formats dominants sur YouTube, dont les contenus sont souvent incarnés par des individus reconnus comme des « influenceurs ». De même, le fonctionnement collectif des lieux autogérés rejette toute forme de personnification du mouvement. Sur le numérique, cette valeur se heurte au fonctionnement des plateformes qui nécessitent une authentification via un compte pour s'y connecter. Même si la gestion peut être collective, il est rare de ne jamais y confier des données personnelles. Par exemple, l'utilisation de YouTube est soumise à l'usage d'un compte Google qui permet facilement de remonter jusqu'à l'individu. Au-delà de la personnification, c'est la responsabilité légale des chaînes 
YouTube qui est questionnée. La vidéo en ligne souligne donc la nécessité de penser les implications politiques du partage de contenus sur les plateformes numériques.

\section{Corpus étudié}

- À Bure-les-Bains, on boit Lejuc sans modération : https ://vimeo.com/194173016

- Bure, bataille pour le bois Lejuc (Teaser 1) : https://www.youtube.com/watch? v=PNZlyvoYGX8

- Bure, bataille pour le bois Lejuc (Teaser 2) : https://www.youtube.com/watch? v=7Vokw1eZTww

- Marche aux flambeaux vers l'Andra ! : https ://www.youtube.com/watch?v=ztBOPVnMAZs

-Bure: les grilles de l'Andra sont tombées!: https://www.youtube.com/watch? $\mathrm{v}=\mathrm{u} 23$ hqajNbBo

- Un ingénieur de l'Andra verse de l'essence sur les opposant-es à Bure: https:// www.youtube.com/watch?v=kzit6sjjt-E

- Un employé de l'Andra verse de l'essence sur des opposants à Cigéo: https:// www.youtube.com/watch?v=s1AdSRO1fIA

-[Détournement] Gérard Longuet «Je suis l'élu du nucléaire » : https ://www.youtube.com/ watch?v=TpZ-gGxE_T8

- Bogues ! : https ://www.youtube.com/watch?v=P5UVHaWDbfQ

- Chaîne «Paroles de malfaiteur-e-s »: https://www.youtube.com/channel/ UCQMCWPAOOis_6QVYtdUf5cg

- Bure été 2016-automne 2017 : https ://vimeo.com/246437223

- Défendre le bois Lejuc, bloquer CIGEO ! : https ://www.youtube.com/watch?v=lUTAPLhCIyw

-J'ai approché des déchets radioactifs : https ://www.youtube.com/watch?v=ck7vKZ5hq3k

- Comment préparer le futur? https ://www.youtube.com/watch?v=IBjRAFGT_OE

- Qu'est-ce qu'il y a après le nucléaire ? https ://www.youtube.com/watch?v=ZjES_CTOb_I

-L'énergie du futur de ta grand-mère https://www.youtube.com/watch?v=3PHfC4ynj58 (vidéo non accessible)

-Vos factures EDF vont exploser: le vrai coût du nucléaire https ://www.youtube.com/ watch?v=09uAyKSpPwI

- Exploration de Tchernobyl dans la zone radioactive https://www.youtube.com/watch? $\mathrm{v}=$ WXh_wArodNk

- Dans les zones radioactives de Fukushima https://www.youtube.com/watch? v=MCVWDaO8gmg

- Et tout le monde s'en fout : les déchets https ://www.youtube.com/watch?v=BqyUne1vSSs

\section{BIBLIOGRAPHIE}

Allard, L. (2007). « Émergence des cultures expressives », d'Internet au mobile. Médiamorphoses. (21), 19-25. Disponible :http://documents.irevues.inist.fr/handle/2042/23558 (Accessed : 29 Nov 2019). 
d'Allens, G. et Fuori, A. (2017). Bure, la bataille du nucléaire. Paris : Éd. du Seuil.

Andra. (2019). Inventaire national des matières et déchets radioactifs 2019. ChâtenayMalabry :Andra.Available at: https ://inventaire.andra.fr/sites/default/files/documents/pdf/fr/ inventaire_national-essentiels-2019.pdf(Accessed : 29 Nov 2019).

Andrejevic, M. (2009). 'Exploiting YouTube : Contradictions of User-Generated Labor', in Pelle Snickars et Patrick Vonderau (eds.) The YouTube reader. Mediehistoriskt arkiv. Stockholm : National Library of Sweden. pp. 406-423.

Babeau, F. (2014). « La participation politique des citoyens 'ordinaires' sur l'Internet. La plateforme YouTube comme lieu d'observation ». Politiques de communication. 2 (3), 125-150.

Badouard, R. (2013). « Les mobilisations de clavier ». Réseaux. 5 (181), 87-117.

Ballarini, L., Costantini, S. et Kaiser, M. (eds.) (2019) Financement participatif : les nouveaux territoires du capitalisme. Questions de communication Série Actes. Nancy : PUN - Éditions universitaires de Lorraine.

Balleys, C. (2019). «L'intimité militante sur Youtube : la visibilité médiatique au service de la libération sexuelle ». Questions de communication. 35 (1), 125-136.

Blondiaux, L. (2008). « Démocratie délibérative vs. démocratie agonistique ? Le statut du conflit dans les théories et les pratiques de participation contemporaines ». Raisons politiques. 30 (2), 131-147.

Bonaccorsi, J. (2013) . "Approches sémiologiques du Web », in Christine Barats (ed.) Manuel d'analyse du Web. Paris : Armand Colin. 125-146.

Bourdeloie, H. et Chevret-Castellani, C. (2019). L'impossible patrimoine numérique ? Mémoire et traces. Paris : Le Bord de l'eau.

Bullich, V. (2015). « Régulation des pratiques amateurs et accompagnement de la professionnalisation : la stratégie de YouTube dans la course aux contenus exclusifs ». Les Enjeux de l'information et de la communication. (16/3B), 27-42.

Candel, E. et Gomez-Mejia, G. (2017). « Le bouton like : poétique du clic, vertige des discours ». Semen. (42).

Cardon, D. et Granjon, F. (2013). Médiactivistes. Paris : Presses de Sciences Po.

Carlino, V. (2019). L'énergie de la contestation : formes de désaccord et arènes du conflit sur le nucléaire en Lorraine. Thèse en sciences de l'information et de la communication. Metz : Université de Lorraine. Available at: https ://hal.univ-lorraine.fr/tel-02090208 (Accessed : 29 Nov 2019).

Costanza-Chock, S. (2012). "Mic Check! Media Cultures and the Occupy Movement”. Social Movement Studies. 11 (3-4), 375-385.

Cuny, C. et Nez, H. (2013). « La photographie et le film : des instruments de pouvoir ambivalents ». Participations. 7 (3), 7-46.

Dechézelles, S. (2017). « Une ZAD peut en cacher d'autres. De la fragilité du mode d'action occupationnel ». Politix. (117), 91-116.

Dias da Silva, P. (2015). « La vidéo en ligne comme outil de communication politique en Europe ». Communication et langages. 1 (183), 59-81.

Faris, D.M. (2012). «La révolte en réseau : le « printemps arabe » et les médias sociaux ». Politique étrangère. Printemps (1), 99-109. 
Flichy, P. (2010). Le sacre de l'amateur. Paris : Éd. du Seuil.

Fraser, N. (2001) « Repenser la sphère publique : une contribution à la critique de la démocratie telle qu'elle existe réellement ». Loïc Blondiaux (ed.). Hermès. 3 (31), 125-156.

Frau-Meigs, D. (2017). « Les Youtubeurs : les nouveaux influenceurs !» Nectart. 5 (2), 126-136.

Granjon, F. (2003). « Les militants-internautes. Passeurs, filtreurs et interprètes ».

Communication. Information médias théories pratiques. 1 (22), 11-32.

Granjon, F., Papa, V. et Tuncel, G. (2017) Mobilisations numériques : politiques du conflit et technologies médiatiques. Paris : Presses des Mines.

Kim, J. (2012) « The institutionalization of YouTube : From user-generated content to professionally generated content« . Media, Culture et Society. 34 (1), 53-67.

Les Temps Modernes (2016). Nuit Debout et notre monde. 691 (5). Paris : Gallimard.

Lussault, M. (2017). Hyper-lieux : les nouvelles géographies politiques de la mondialisation. Paris, France : Éditions du Seuil.

Mabi, C. (2016). « Luttes sociales et environnementales à l'épreuve du numérique : radicalité politique et circulation des discours ». Études de communication. langages, information, médiations. (47), 111-130.

Mabi, C. et Gruson-Daniel, C. (2018). « Formes et mouvements politiques à l'ère numérique ». RESET. Recherches en sciences sociales sur Internet. (7)

Mann, S. et Ferenbok, J. (2013). « New Media and the power politics of sousveillance in a surveillance-dominated world ». Surveillance et Society. 11 (1/2), 18-34.

Negt, O. (1972) L'espace public oppositionnel. Paris : Payot.

Neumann, A. (2018). Negt (Oskar). Publictionnaire. Dictionnaire encyclopédique et critique des publics. Available at: http://publictionnaire.huma-num.fr/notice/negt-oskar (Accessed : 29 Nov 2019).

Ollitrault, S. (1996). « Science et militantisme : les transformations d'un échange circulaire. Le cas de l'écologie française ». Politix. 9 (36), 141-162.

Patinaux, L. (2017). Enfouir des déchets nucléaires dans un monde conflictuel. Une histoire de la démonstration de sûreté de projets de stockage géologique, en France (1982-2013). Thèse de doctorat en histoire. Paris : EHESS. Disponible :https ://hal.archives-ouvertes.fr/tel-01974327 (Accessed : 29 Nov 2019).

Rey, N. (2018). « Faire du cinéma comme on occupe des zones à défendre », jef klak, 16 Jan. Available at: https ://www.jefklak.org/faire-cinema-on-occupe-zones-a-defendre (Accessed : 29 Nov 2019).

Sebbah, B. (ed.) (2017). L'événement politique en ligne. Sciences de la société (102). Toulouse : Presses universitaires du Midi.Sedda, P. (2015). «L'Internet contestataire. Comme pratique d'émancipation. Des médias alternatifs à la mobilisation numérique ». Les Cahiers du numérique. $11(4), 25-52$.

Smyrnaios, N. (2017). Les GAFAM contre l'Internet : une économie politique du numérique. Brysur-Marne : INA éditions.

Snickars, P. et Vonderau, P. (eds.) (2009). The YouTube reader. Mediehistoriskt arkiv (12). Stockholm : National Library of Sweden. 
Subra, P. (2017). De Notre-Dame-des-Landes à Bure, la folle décennie des « zones à défendre » (2008-2017). Hérodote. (165), 11-30.

Theviot, A. (2018). Facebook, Twitter, YouTube. Vers une « révolution » de la participation et de l'engagement politique en ligne ? Idées économiques et sociales. 4 (194), 24-33.

Tilly, C. (1986). La France conteste : de 1600 à nos jours. Paris : Fayard.

Topçu, S. (2006). Nucléaire : de l'engagement « savant » aux contre-expertises associatives. Natures Sciences Sociétés. 3 (14), 249-256.

Towner, T.L. et Muñoz, C.L. (2018). « Picture Perfect? The Role of Instagram in Issue Agenda Setting During the 2016 Presidential Primary Campaign ». Social Science Computer Review. 36 (4), 484-499.

Wamé, B. (2018). « Réseaux sociaux numériques et minorité ». Les Cahiers du numérique. 14 (3), 107-127.

Yanoshevsky, G. (2009). «L'usage des vidéoblogs dans l'élection présidentielle de 2007. Vers une image plurigérée des candidats ». Mots. Les langages du politique. (89), 57-68.

Young, I.M. (2002). Inclusion and Democracy. New York : Oxford University Press.

Zannettou, S., Chatzis, S., Papadamou, K. et Sirivianos, M. (2018). « The Good, the Bad and the Bait: Detecting and Characterizing Clickbait on YouTube' ", in 2018 IEEE Security and Privacy Workshops (SPW). May 2018, 63-69.

\section{NOTES}

1. https ://www.youtube.com/user/ANONIMALOfficiel

2. https ://www.youtube.com/user/histoirebreve

3. https://www.youtube.com/lejeuvideal

4. Les personnes interrogées ont accordé leur participation à ces entretiens, ainsi que leur enregistrement. En raison de l'éloignement géographique et des procédures judiciaires dont ils font l'objet, ils ont souhaité échanger par appels téléphoniques via une messagerie sécurisée. En retour, nous nous sommes engagés à respecter leur anonymat ainsi qu'à ne pas diffuser les enregistrements.

5. CNDP (2013). Les objectifs, modalités et principes du débat. Accessible à : http:// cpdp.debatpublic.fr/cpdp-cigeo/debat/debat_public.html (consulté le 28/5/2019).

6. Empruntée à Charles Tilly (1986, p.541-542), la notion désigne les «moyens d'agir en commun sur la base d'intérêts partagés " propres à certaines mobilisations sociales.

7. Source : L’Automédia, https ://vmc.camp/ (archive personnelle)

8. Même si ces dernières soulèvent des questions essentielles que nous aborderons dans la partie Critique et intégration des normes des plateformes.

9. À la fin du mois de juillet 2016, l'Andra construit un mur pour tenir les militants à l'écart de la zone de travaux du bois Lejuc. Jugeant cette action illégale, les militants décident de l'abattre et inscrivent des slogans sur les dalles qui le composent. Une peine de prison avec sursis a été requise contre deux militants et l'Andra a été condamnée à remettre la forêt en état.

10. L'acronyme signifie « zone à défendre ». 
11. Le collectif «Les scotcheuses » spécialisé dans le super 8 s'est rendu à Bure pour tourner un «film d'anticipation post-catastrophique ». Dans une interview accordée au média jef klak, le collectif souligne que le super 8 permet de développer un autre regard sur le réel grâce à l'analogique (Rey, 2018). Alors que le super 8 était un matériel léger et peu coûteux pendant mai 1968, il acquiert aujourd'hui une forme de rareté face à l'abondance des possibilités de prise de vue des smartphones.

12. Archive disponible sur : https ://www.youtube.com/watch?v=s1AdSRO1fIA.

13. La plateforme de formation "Creators Academy" de YouTube insiste sur l'importance du marketing de contenu dès la première vidéo d'initiation : «La miniature est très importante car c'est comme une mini-publicité de votre vidéo et tout le monde ne lit pas le titre.» Source: YouTube (n.d.) Principes de base de YouTube. Accès: https:// creatoracademy.youtube.com/page/lesson/subscriber-advantage?hl=fr (consulté le 28/5/2019).

14. L'Andra a notamment conclu des partenariats avec les chaînes YouTube Anonimal, Dave Sheik et Simon Puech. Avec les subventions et le financement participatif (Ballarini et al., 2019), le partenariat constitue un moyen de financer la création sur YouTube.

15. Ceci donne une dimension sémiotique supplémentaire aux contenus. L'écart entre les stratégies de promotion et le contenu effectif des vidéos peut avoir une incidence sur la perception du créateur. Ceci s'observe notamment à travers l'utilisation de titres particulièrement aguicheurs qui peuvent être perçus négativement (Zannettou et al., 2018).

16. Vimeo et YouTube constituent les deux plateformes principalement utilisées pour la vidéo en ligne.

\section{RÉSUMÉS}

La vidéo en ligne est largement utilisée dans les mouvements de contestation. Les réseaux sociaux numériques permettent de diffuser les manifestations en direct depuis des smartphones. L'usage de la vidéo par des groupes militants marginalisés reste peu explorés. À partir du terrain de la contestation antinucléaire radicale, cet article entend contribuer à ce champ de recherche. La vidéo est considérée comme un moyen pour les militants marginalisés de participer au débat public. La réflexion montre que le potentiel mobilisateur des technologies doit être questionné. En effet, les militants envisagent la vidéo comme étant intégrée à la lutte sociale et politique. Ce faisant, ils se heurtent aux valeurs et aux normes de production de contenu des plateformes numériques de diffusion, notamment YouTube. La vidéo souligne alors les limites de l'engagement en ligne et son articulation nécessaire aux modes d'action traditionnels.

There is a widespread use of online videos by social movements. Protest is now broadcasted live from smartphones. However, marginalized activists groups' use of video needs more research. This article contributes to this field of research by analyzing radical antinuclear activism. Video is seen as a way to take part in the public debate. The study shows that mobilization through technology must be questioned. Indeed, video appears to play an important role in the social and 
political struggle. It also shows that there is a conflict between activists's objectives and the values of online video sharing platform's values, such as YouTube. Video highlights online activism limitations and its key role in connecting to offline practices.

INDEX

Mots-clés : médiactivisme, plateformes, radicalité, vidéo, YouTube

Keywords : mediactivism, platforms, radicalness, video, YouTube

\section{AUTEUR}

\section{VINCENT CARLINO}

Post-doctorant en sciences de l'information et de la communication

Université de Neuchâtel, Académie du journalisme et des médias, CH-2000 Neuchâtel, Suisse, vincent.carlino@unine.ch

Université de Lorraine, Centre de recherche sur les médiations, F-57000 Metz, France 